\title{
南北差异视角下个体食物浪费行为 基于大学生高校食堂就餐情境的分析
}

\author{
钱 龙 $^{1}$, 李 丰 $^{1}$, 钱 壮 $^{1}$, 王灵恩 ${ }^{2}$
}

(1. 南京财经大学粮食安全与战略研究中心, 南京 210003; 2. 中国科学院地理科学与资源研究所, 北京 100101)

\begin{abstract}
摘要: 饮食习惯一直被认为是造成食物浪费的重要因素。基于对 29 省 29 所高校 9192 份大学 生食堂就餐的实地调查问卷, 从南北差异视角探讨了个体食物浪费行为的差异特征。研究表 明: 相对北方籍大学生, 南方籍大学生浪费食物的概率更高, 南方籍大学生在高校食堂就餐时 食物浪费量和浪费率也高于北方籍大学生。通过中介效应模型, 证实南北方主流饮食模式是 引发浪费差异的关键所在。进一步匹配大学生南北方籍贯和所就读高校的南北区位, 并以“南 方大学生在南方高校求学” 模式为基准, 发现 “南方大学生到北方高校求学”模式下, 个体有更 低的食物浪费概率、浪费量和浪费率。但“北方大学生到南方高校求学”模式下, 个体没有显示 出较低的食物浪费概率、食物浪费量和食物浪费率, 从而稳健地证实南北方主流饮食模式是引 发浪费差异的关键所在这一结论。
\end{abstract}

关键词: 食物浪费; 高校食堂; 南北差异; 主流饮食模式

2018 年 10 月 16 日，第38个世界粮食日主题是 “努力实现零饥饿”。尽管已经迈人了 21 世纪，但是饥饿仍像幽灵一样飘荡在人类社会。据联合国粮农组织、联合国世界粮食 计划署和欧盟联合发布的《全球粮食危机报告 2018》显示，2017年全球有 51 个国家约 1.24 亿人受到急性粮食不安全的影响 ${ }^{[1]}$ 。联合国 2018 年发布的《世界粮食安全和营养状 况》也指出，2017年全球面临食物不足困境的人口约 8.21 亿，相当于全世界每 9 个人中 就有 1 个人受到饥饿的威胁 ${ }^{[2]}$ 。然而，在饥饿肆虐的同时，全世界却有大量的食物被白白

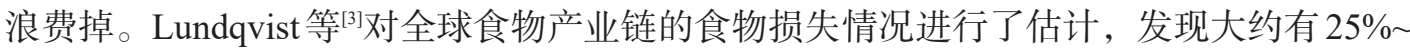
$50 \%$ 的食物被损耗掉。联合国粮农组织（FAO）2011年发布的报告称，全球生产和制造 的食物中，大约有 $13 \times 10^{8} \mathrm{t}$ 被浪费，约占当年全球食物总产量的 $32 \%{ }^{\left[{ }^{[4]}\right.}$ 。如果这些食物不 被损耗，而是被有效利用起来，则足以充分供给全球的饥饿人口，实现世界 “零饥饿” 的美好愿望或许能成为现实。

食物浪费发生在食物供应链的各个环节 ${ }^{[5]}$ 。其中，收获、干燥、储藏、运输、加工、 销售等流通过程发生的浪费属于技术层面的损耗 ${ }^{[6]}$ ，是一种相对客观的食物损失。而消费 终端的食物浪费则更多是人为引起，多属于人们主观行为引致的浪费。相对而言，流通 阶段的食物浪费容易得到解决，这部分食物损耗可以通过技术、物流与设备水平的创新

收稿日期：2019-09-12；修订日期：2019-11-20

基金项目：国家自然科学基金项目（71803077，41701620); 国家公益性行业科研专项（201513004）；教育部人文社 会科学研究基金项目 (18YJC90128)

作者简介：钱龙（1988- ), 男, 安徽枞阳人, 博士, 副教授, 研究方向为食物经济。E-mail: qianlongy101@126.com 通讯作者：王灵恩 (1987- ), 男, 山东沂南人, 博士, 副研究员, 研究方向为区域发展与可持续消费。

E-mail: wangle@igsnrr.ac.cn 
与升级予以缓解。消费终端食物浪费则更难遏制，人类决策的复杂性决定了减少这一 层面的食物浪费面临诸多困难 ${ }^{[7]}$ 。也正因如此，近年来，食物浪费的驱动因素 ${ }^{[8-10]}$, 以 及如何才能有效缓解或减少食物浪费 ${ }^{[1-14]}$ 引起越来越多的学者关注。有较多文献对居民 家庭的食物浪费现象进行了探讨, 发现居民家庭食物浪费行为受到多重因素的影响, 包 括个体特征（如性别、年龄、文化程度等）、对食物浪费的认知和观念 ${ }^{[15,16]}$ 、消费习惯等 等 ${ }^{[17]}$, 以及家庭层面特征，如家庭人口规模 ${ }^{[18]}$ 、人口结构 ${ }^{[19]}$ 、家庭经济水平 ${ }^{[20]}$ 、家庭对 食物保鲜设备的科学运用 ${ }^{[21]}$ 等等。随着居民在外消费的日益普遍，餐饮服务业的食物浪 费现象也得到较多关注 ${ }^{[22]}$ 。已有文献发现，除个体特征、家庭特征外，餐饮质量、就餐 原因、面子文化、信息干预等因素也可能影响到居民在外就餐的食物浪费行为 ${ }^{[9,13,23]}$ 。关 于其他场所食物浪费也有部分文献涉及，比如 Marlette 等 ${ }^{[24}$ 和 Adams 等 ${ }^{[25]}$ 对中小学食堂 食物浪费进行了调查, Buzby等 ${ }^{[26]}$ 、Moult 等 ${ }^{[2]}$ 对超市和零售业食物浪费的研究, Barton 等 ${ }^{[28]}$ 和Zakiah 等 ${ }^{[29]}$ 对医院病人食物浪费行为的分析, Whitehar等 ${ }^{[30]}$ 、Scherhaufer 等 ${ }^{[31]}$ 对大 学食堂食物浪费的调查, 等等。

尽管食物浪费更多发生在发达国家和地区，但是作为发展中国家的中国，同样存 在较为严重的食物浪费。近年来，食物浪费现象得到国内部分学者的关注，初步形成 一批有影响力的成果。一些研究对食物浪费规模进行了测算, 如中国农业大学的一项 研究表明，2006-2008 年中国人浪费的食物总量足以养活 2.5 亿 3 亿人 ${ }^{\mathbb{1}}$ 。胡越等 ${ }^{[32]}$ 利 用 GTAP 模型测算中国食物浪费量, 估算出中国一年的粮食浪费量为 1.2 亿 $\mathrm{t}$, 相当于浪 费了 2.76 亿亩播种面积和 316.1 亿 $\mathrm{m}^{3}$ 农业用水。来自中国科学院地理科学与资源研究所 的一项研究表明，中国 2015 年在餐桌上浪费的粮食高达 1700 万 1800 万 $\mathrm{t}$ ，相当于 3000 万 5000 万人一年的食物量 ${ }^{(2)}$ 。江金启等 ${ }^{[10]}$ 基于中国健康与营养调查，推算出 2016 年中国居民家庭全年食物浪费总量高达 1055.60 万 1501.55 万 $\mathrm{t}$ ，相当于当年 $4.47 \%$ $\sim 5.2 \%$ 的粮食被白白浪费。另外，还有一些文献涉及食物浪费导致的负面资源环境效 应。如 Song 等 ${ }^{[18}$ 基于 CHNS 数据, 分析了中国居民食物浪费带来的碳足迹和水足迹。 Wang 等 ${ }^{[33}$ 基于拉萨市餐饮业食物消费的调查, 对食物浪费的资源环境成本进行了定量 核算。张丹等 ${ }^{[3436}$ 基于北京市餐饮机构的调查，分析了餐馆食物浪费的氮足迹、碳足迹 和磷足迹。但整体而言, 国内关于食物浪费的研究仍然较少, 且既有文献视角偏宏 观，基于微观视角分析个体浪费行为的成果还不多见 ${ }^{[13,14]}$ 。

公共食堂作为大多数居民日常就餐的主要形式, 日益引起国外学者的关注。如Painter 等 ${ }^{[3]}$ 对南非 Rhodes 大学 205 名大学生的问卷调查, Pinto 等 ${ }^{[38}$ 对葡萄牙 Lisbon 大学农学 院餐厅持续一个月的追踪调查, Lorenz 等 ${ }^{\left[{ }^{3} 9\right.}$ 对德国一所大学 343 名大学生的调查, Schaubroeck 等 ${ }^{[3]}$ 以比利时 Ghent大学为对象进行的案例研究等。中国高校食堂中的食物 浪费十分惊人。有诸多媒体曾对中国高校食堂的食物浪费现象进行深度采访, 并感叹青 年大学生不懂得 “粒粒皆辛苦”，不珍惜宝贵的粮食。例如人民网 2013 年的调查 ${ }^{3}$ 、央视

(1) 数据来源于《政协常委武维华: 中国每年浪费的食物能养活3亿人》, 2010-3-10, http://www.chinanews.com/ gn/news/2010/03-10/2161052.shtml。

(2) 数据来源于 《中国食物浪费量约为每年 1700 万至 1800 万 $\mathrm{t}\rangle, 2016-11-29$, http://www. igsnrr.ac.cn/xwzx / xwdt_cmsm/201611/ t20161129_4711392.html。

(3) 数据来源于《调查高校食堂浪费, 受访学生谈剩菜原因》, 2013-11-21, http://edu.people.com.cn/n/2013/1121/ c 1053-23611050.html。 
网 2017 年的报道 ${ }^{4}$ 等等。然而，当前还鲜有严谨的研究聚焦中国高校食堂场所的食物浪 费。据国家统计局公开发布的资料显示，2017年中国已经拥有各类高校2914所，在校大 学生数量达到 3753.5 万人, 位居世界第一。因此, 对中国高校的食堂浪费进行调查, 分 析青年大学生食堂就餐的浪费行为及其影响因素，不仅有助于揭开高校食堂场所食物浪 费的黑箱，而且有助于针对性地出台政策减少高校食堂领域的食物浪费规模。

本文并不打算从个体特征、家庭特征、餐饮特征等传统视角研究中国高校食堂的食 物浪费现象，而是选择从南北差异视角分析个体的食物浪费行为。正如一些跨国研究所 发现的那样 ${ }^{[40]}$, 不同国度的消费习惯、饮食文化有较大差异，不同饮食模式和饮食文化 导致不同国家居民的食物浪费行为有较大差异 ${ }^{[15,16,41]}$ 。在研究中国高校食堂的大学生食物 浪费行为时, 很可能也需要考虑地域饮食差异。中国是一个地理面积广䘚的国度，不同 区域人们的餐饮习俗和饮食文化有很大差异。一个经常被提及、且很难被忽视的就是南 方人和北方人的饮食差异。中国北方主要种植小麦，中国南方主要种植水稻，这也造成 了北方人更多以面粉为主食，形成了北方特色的 “粉面” 饮食模式; 而南方人则更多以 米饭为主食, 注重以菜品配合米饭, 形成了南方主流的 “饭菜” 饮食模式 ${ }^{[42]}$ 。同一所大 学的学生来自四面八方，他们的饮食习惯和饮食文化不可避免烙上鲜明的地域特点。鉴 于南北方的主流饮食模式差异明显，因此本文试图探索：在高校食堂就餐时，南方人和 北方人的食物浪费表现有差异么? 南方人和北方人，谁更可能浪费食物，谁浪费了更多 食物，谁的食物浪费程度更严重? 如果南方人和北方人确实存在差异化食物浪费表现， 是不是南北方不同饮食模式引致的呢?

本文后续安排如下：首先是研究设计，主要介绍了调研开展和数据采集的过程，对 特征变量的引人进行了详细说明, 并介绍了相应的研究方法; 其次是实证结果与分析, 包括描述性分析和整体样本的计量分析；再次是作用机制检验和进一步分析，主要是验 证南北方主流饮食模式是否是南方人和北方人差异化食物浪费表现的作用机制; 文章最 后一部分是简要结论与讨论。

\section{1 研究方法与数据来源}

\section{1 数据采集}

2018 年课题组开展了“中国高校食堂食物浪费调查”的调查。在正式问卷调查之 前，分别在江苏和河南的两所高校进行了预调研，并对调查问卷进行了修正和完善。中 国目前有各类高校 2914 所，受限于经济和人力，以及开展大规模食物浪费调查面临的实 际困难, 课题组借助熟人关系网络展开调研。在多方帮助之下, 课题组按照 “1省 1 校” 原则，对除西藏、港澳台外的 30 个省市（自治区）的 30 所高校进行了问卷调查，并在大 多数高校随机采集 300 350 份左右的食堂就餐浪费问卷。从此次抽查的学校类型来看, 既包括生源相对多元化的全国重点大学（985、211 高校)，也包括生源主要来自本地的地 方普通院校，以及居于两者之间的区域性一本招生高校，样本覆盖相对全面。

实际调查由两位调研员协作完成，分别负责询问和采集受访者个体特征，家庭特 征和餐饮特征，并录人纸质问卷。在受访者用餐完毕后，两人相互配合，使用电子秤对

(4) 数据来源于 《舌尖上的浪费: 部分高校食堂和自助餐厅浪费减少》, 2017-5-29, http://news.sina.com.cn/c/ 2017-05-29 /doc-ifyfqqyh8913863.shtml。 
本餐次的食物残留进行称重，从而获得关键的食物浪费数据。由于在河南省的调研受到 干扰, 且获得样本较少, 因此本文没有保留河南高校的数据。最终采用的是对东部 11 个 省、中部 7 个省、西部 11 个省共 29 所高校的调查数据。历时两个多月，在 19 名培训员和 300 多位调查员的共同努力下，课题组累计称重 30000 余次，最终获得了 9192 份有效问 卷。样本的具体分布如表 1 所示。

\section{表 1 样本分布情况}

Table 1 Sample distribution

\begin{tabular}{|c|c|c|c|c|c|c|c|}
\hline 序号 & 省（市、自治区） & 学校 & 样本量/份 & 序号 & 省（市、自治区） & 学校 & 样本量/份 \\
\hline 东部 & & & 3764 & 4 & 湖南 & 湖南大学 & 325 \\
\hline 1 & 上海 & 上海财经大学 & 278 & 5 & 山西 & 山西财经大学 & 306 \\
\hline 2 & 江苏 & 南京邮电大学 & 596 & 6 & 吉林 & 吉林大学 & 360 \\
\hline 3 & 浙江 & 湖州师范学院 & 304 & 7 & 黑龙江 & 哈尔滨工业大学 & 296 \\
\hline 4 & 北京 & 北京师范大学 & 326 & 西部 & & & 3452 \\
\hline 5 & 天津 & 天津财经大学 & 300 & 1 & 陕西 & 陕西师范大学 & 317 \\
\hline 6 & 河北 & 河北大学 & 308 & 2 & 甘肃 & 兰州理工大学 & 330 \\
\hline 7 & 山东 & 山东财经大学 & 300 & 3 & 宁夏 & 宁夏大学 & 300 \\
\hline 8 & 辽宁 & 沈阳理工大学 & 350 & 4 & 青海 & 青海师范大学 & 300 \\
\hline 9 & 广东 & 深圳大学 & 348 & 5 & 内蒙古 & 内蒙古工业大学 & 300 \\
\hline 10 & 福建 & 厦门理工学院 & 331 & 6 & 新疆 & 石河子大学 & 300 \\
\hline 11 & 海南 & 海南大学 & 323 & 7 & 四川 & 四川农业大学 & 329 \\
\hline 中部 & & & 1976 & 8 & 重庆 & 重庆理工大学 & 365 \\
\hline 1 & 安徽 & 宿州学院 & 300 & 9 & 贵州 & 贵州大学 & 309 \\
\hline 2 & 江西 & 江西农业大学 & 298 & 10 & 广西 & 广西大学 & 302 \\
\hline 3 & 湖北 & 武汉轻工大学 & 91 & 11 & 云南 & 西南林业大学 & 300 \\
\hline
\end{tabular}

\section{2 变量定义}

（1）食物浪费行为。食物浪费行为是本文所关注的被解释变量，以往成果多关注是 否有食物浪费现象，或者关注食物浪费量的测度，也有少数文献涉及食物浪费率的测 度, 却少有研究同时使用这三个指标。主要原因是获得准确的食物浪费数据耗时耗力, 在实践操作中十分困难 ${ }^{[43]}$, 导致大多数成果依赖于二手数据。考虑到单一指标难以有效 反映个体的食物浪费行为，本文同时使用上述三个指标来予以测度，从而更为全面地显 示个体在食堂就餐时的食物浪费行为。其一，根据大学生就餐后是否有可食用食物的剩 余，设定一个二分类变量。其二，更为准确的，本文通过剩余称重法，将餐后的可食用 食物进行分类和称重，精确到小数点后 2 位（单位 $\mathrm{g}$ ），加总之后计算出本餐次的食物浪 费量 ${ }^{[44]}$ 。其三, 根据个体实际就餐的品种, 通过换算餐前的标准重量和餐后的剩余重 量, 得出本餐次的食物浪费率 ${ }^{[45]}$ 。其中, 获取指标二和指标三需要经历较为繁杂的清理 和称重过程, 这里参考既有成果, 将餐前和餐后的食物予以分类进行称重 ${ }^{5}$, 并精确到小 数点后 2 位。但一些食物垃圾，如蔬菜皮、豆渣、骨头等不属于食物范畴的不在统计范 围之内 ${ }^{[13]}$ 。此外, 南北方日常饮食中液体食物出现频率有较大差异, 参照以往文献 ${ }^{[30]}$,

(5) 此次调查将食物剩余按照米饭、面食、猪肉、牛羊肉、禽肉、水产品、蛋类、乳制品、豆制品、蔬菜、瓜果 类一共 11 个大类分别进行了称重。 
汤汁等液体也并不在此次食物浪费调查的统计范围中。因此, 相对以往研究, 本文对个 体食物浪费行为的测度更加精确和全面。

（2）南北方籍贯。本文重点关注南方人与北方人的食物浪费行为差异，因此，使用 家庭户籍所在地来识别大学生的南北方籍贯, 设置一个二元变量来显示个体是南方人还 是北方人。南北划分方面, 按照共识, 以秦岭——淮河为南北分界线, 将四川、云南、 重庆、贵州、广西、湖北、湖南、安徽、江苏、上海、浙江、江西、福建、广东、海南等 15 个省（市、自治区）划分为南方，将黑龙江、吉林、辽宁、内蒙古、新疆、甘肃、青 海、宁夏、陕西、山西、河北、北京、天津、山东等 14 个省（市、自治区）划分为北方。

（3）其他控制变量。个体的食物浪费决策十分复杂, 受到多个层面因素的影响 ${ }^{[4] 。}$ 参考已有文献，本文引人个体特征维度、家庭特征维度、餐饮特征维度和区域特征维度 4 个层面的控制变量。具体如下:

个体特征层面。本文引人了性别、年龄、学历、 $B M I$ 指数、是否独生子女、民族、 接触节粮宣传的频率一共 7 个控制变量。以往研究表明，不同性别、年龄、文化水平和 体格特征的个体，其食物浪费行为可能存在有一定差异 ${ }^{[1,22,47]}$, 因此予以控制。一个流行 的认知是, 相对于非独生子女, 独生子女更可能浪费食物 ${ }^{[10]}$, 因而引人是否独生子女这 一虚拟变量。除上述客观特征外，考虑到不同民族的文化背景差异较大，这可能影响到 个体的食物浪费决策 ${ }^{[48]}$, 因而也予以引人。近年来，通过信息干预来减少食物浪费，引 起了越来越多的学者重视。一些研究支持, 有效的信息干预能够减少食物浪费 ${ }^{[13]}$; 但也 有一些研究发现，信息干预并不能有效减少食物浪费 ${ }^{[49}$ 。考虑到中国高校的实际情况， 本文引人个体接触节粮宣传的频率来识别信息干预所发挥的作用。

家庭特征维度。本文引人家庭人口规模、家庭经济水平这 2 个控制变量。虽然并非 针对食堂就餐中出现的浪费行为, 但关于居民家庭和餐馆消费的食物浪费研究证实, 家 庭人口规模可能会对个体食物浪费行为有影响 ${ }^{[00]}$ 。因此, 本文也予以控制。家庭富裕程 度被较多的文献证实很可能对个体的食物浪费行为有影响 ${ }^{[20]}$, 因而, 本文也引人这一变 量来控制家庭经济条件可能发挥的作用。

餐饮特征层面。本文引人了餐次、就餐持续时间、一同就餐人数、饭菜重量、餐盘 类型、对饭菜口味的满意度一共 6 个相关控制变量。之所以引入餐次、就餐持续时间和 一同就餐人数是考虑到大学生日常饮食规律和公共场所就餐特点可能发挥的影响。产生 食物浪费的一个重要来源是超标准食物供给, 因此引人餐前饭菜重量这一变量 ${ }^{[9,51]}$ 。且以 往研究发现, 食堂提供的配餐工具可能会影响到食物浪费 ${ }^{[24]}$ 。因此, 本文也予以引人。 除上述客观特征，还不能忽视个体对餐饮质量主观评价所发挥的作用。通常而言，个体 对餐饮口味的满意度越高，所产生的食物浪费会越少 ${ }^{[23]}$ 。

此外，鉴于中国地域广大，因此不能忽视区域异质性这一客观事实。其中一个显而 易见的表现是，东部、中部和西部的经济社会发展有明显的层次性。有鉴于此，本文引 人了是否东部和是否中部两个虚拟变量, 来控制这一层面因素所发挥的作用。

上述变量的定义和相应的描述统计性分析如表 2 所示。

\section{3 模型选择}

为有效测度南北方籍贯对个体食物浪费行为的影响，借鉴已有文献，将基准模型设 定如下: 
表 2 变量定义及描述性分析

Table 2 Variable definition and descriptive analysis

\begin{tabular}{|c|c|c|c|c|}
\hline 变量名称 & 变量定义 & 观测值/个 & 均值 & 标准差 \\
\hline 食物浪费行为 & 本餐次是否有浪费： $1=$ 有； $0=$ 没有 & 9128 & 0.74 & 0.44 \\
\hline 食物浪费量 & 本餐次各类食物剩余重量加总/g & 9128 & 61.03 & 73.71 \\
\hline 食物浪费率 & $\begin{array}{l}\text { 本餐次各类食物剩余重量加总 } \times 100 / \text { 此次就餐各类食物 } \\
\text { 的标准重量加总 } / \%\end{array}$ & 9121 & 12.13 & 14.55 \\
\hline 南北方籍贯 & 被调查人家庭所在地：1=南方；0=北方 & 9157 & 0.54 & 0.50 \\
\hline 性别 & $1=$ 男；0=女 & 9191 & 0.52 & 0.50 \\
\hline 年龄 & 岁 岁 & 9169 & 21.25 & 2.33 \\
\hline 学历 & $1=$ 研究生; $0=$ 本科生 & 9169 & 0.19 & 0.39 \\
\hline$B M I$ 指数 & $B M I=$ 公斤数体重 $/$ 身高米数的平方 & 9161 & 20.68 & 2.81 \\
\hline 是否独生子女 & $1=$ 是独生子女; $0=$ 不是独生子女 & 9161 & 0.43 & 0.49 \\
\hline 民族 & $1=$ 汉族；0=少数民族 & 9146 & 0.90 & 0.30 \\
\hline 接触节粮宣传的频率 & $1=$ 较多接触; $0=$ 较少接触 & 9158 & 0.41 & 0.49 \\
\hline 家庭人口规模 & $1=3$ 人及以下; $2=4 \sim 6$ 人; $3=7$ 人及以上 & 8564 & 1.67 & 0.53 \\
\hline 家庭经济水平 & $\begin{array}{l}\text { 学生每月生活费支出/元：1= } \leqslant 1000 ; 2=1000 ~ 1500 ; \\
3=>1500\end{array}$ & 9126 & 1.74 & 0.76 \\
\hline 餐次 & $1=$ 午餐； $0=$ 晚餐 & 9099 & 0.55 & 0.50 \\
\hline 就餐持续时间 & 此次就餐持续多久/分钟 & 9164 & 1.55 & 0.57 \\
\hline 一同就餐人数 & 和被调查人一起来食堂就餐人数/人 & 8473 & 1.89 & 0.78 \\
\hline 餐前饭菜重量 & 被调查人此次就餐饭菜重量/g & 8511 & 524.54 & 155.38 \\
\hline 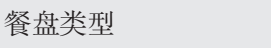 & 1=合成餐盘；0=分装餐盘 & 8972 & 0.66 & 0.47 \\
\hline 饭菜口味满意度 & $3=$ 较为满意; $2=$ 一般满意; $1=$ 不太满意 & 8486 & 2.40 & 0.59 \\
\hline 是否东部 & 高校所在区位：1=东部；0=非东部 & 9192 & 0.41 & 0.49 \\
\hline 是否中部 & 高校所在区位：1=中部；0=非中部 & 9192 & 0.21 & 0.41 \\
\hline 南北方区位 & 高校所在区位：1=南方；0=北方 & 9192 & 0.49 & 0.50 \\
\hline
\end{tabular}

$$
\text { Food }- \text { waste }=\beta_{0}+\beta_{1} S-N+\sum g_{i} X_{i}+\varepsilon_{i}
$$

式中: Food-waste指示大学生的食物浪费行为, 本文分别使用本餐次是否有食物浪费现 象、餐次食物浪费量和餐次食物浪费率三个指标来予以指示; $\beta_{0}$ 表示常数; $\beta_{1}$ 表示判断 南北方籍贯对大学生食物浪费行为的影响; $S-N$ 是本文的核心解释变量, 表示个体是南 方人还是北方人; $g_{i}$ 表示控制变量相应的影响系数; $X_{i}$ 表示一系列控制变量； $\varepsilon_{i}$ 为随机误 差项。具体模型方面，由于个体是否有食物浪费行为是二分类变量，因而选择二元Probit 模型予以分析。对于食物浪费量和食物浪费率，本文则使用Tobit模型分析南北方籍贯对 个体食物浪费量和食物浪费率的影响。

\section{2 结果分析}

\section{1 描述性分析}

为直观显示南方人与北方人的食物浪费行为差异，本文首先进行简单的分组对比分 析（表 3 )。结果显示，南方人和北方人出现食物浪费现象的几率相差不多，南方人仅仅 比北方人高 1.21 个百分点。但每人每餐的食物浪费量和食物浪费率方面，南北方人的差 别较大。南方人每人每餐食物浪费量比北方人高 $12.98 \mathrm{~g}$, 每人每餐食物浪费率高 2.95 个 
百分点。因而，统计描述性分析初步

表 3 描述性分析

表明, 在高校食堂就餐时, 南方人比

北方人更可能出现食物浪费，浪费的 食物更多，食物浪费程度更重。

\section{2 计量模型分析}

为了进一步验证南北方籍贯对大

Table 3 Descriptive analysis

\begin{tabular}{lccc}
\hline 分组 & $\begin{array}{c}\text { 是否有 } \\
\text { 食物浪费 } / \%\end{array}$ & $\begin{array}{c}\text { 每人每餐 } \\
\text { 食物浪费量 } / g\end{array}$ & $\begin{array}{c}\text { 每人每餐 } \\
\text { 食物浪费率 } / \%\end{array}$ \\
\hline 南方人 & 74.36 & 67.08 & 13.51 \\
北方人 & 73.15 & 54.10 & 10.56 \\
\hline
\end{tabular}
学生食物浪费行为的影响, 按照基准模型, 表 4 进行了相应的计量模型分析。在实证分 析之前, 首先进行了多重共线性检验, 结果显示, 所有变量的 $V I F$ 值都小于 10 , 不存在 严重共线性问题。研究结果表明（表 4)，南北方籍贯分别在 5\%、1\%和 $1 \%$ 的显著性水平 正向促进个体是否有食物浪费、本餐次的食物浪费量和本餐次的食物浪费率。即相对于 北方人，南方人确实更可能出现食物浪费，在高校食堂就餐时浪费的更多，浪费程度更 重。通过计算边际效应，发现相对于北方人，南方人出现食物浪费的概率平均要高 2.05 个百分点, 平均每餐食物浪费量会多 $17.10 \mathrm{~g}$, 平均每餐的食物浪费率要高 3.45 个百 分点。这也意味着，在高校食堂就餐时，南方籍学生确实比北方籍学生更浪费食物。

控制变量方面，个体特征层面的性别、学历层次、BMI、接触节粮宣传的频率 4 个特 征变量通过了显著性检验。具体表现为，相对于男性，女性更可能出现食物浪费现象， 本餐次食物浪费量和本餐次食物浪费率也更高。说明大学生在高校食堂就餐时, 女性更 浪费食物 ${ }^{[7]}$ 。学历方面, 相对于本科生, 研究生有着更低的食物浪费概率、食物浪费量 和食物浪费率也更低，表明更高的文化水平有助于减少食物浪费。BMI指数始终在 $1 \%$ 的 显著性水平负向影响个体是否有食物浪费行为、本餐次的食物浪费量和食物浪费率，表 明个体的 $B M I$ 指数越高, 越有助于减少食物浪费，这与理论预期相符。接触节粮宣传的 频率对个体食物浪费行为也有显著影响, 相对于较少接触节粮宣传的个体, 较多接触节 粮宣传的个体出现食物浪费现象的概率更低，食物浪费量更少，食物浪费率也更低。这 意味着信息干预有助于减少食物浪费，这与以往的研究保持一致 ${ }^{[13]}$ 。年龄、是否独生子 女和民族 3 个变量始终没有通过显著性检验，表明年龄不是影响个体食物浪费的关键因 素, 独生子女也没有比非独生子女表现的更浪费食物, 汉族与少数民族在食物浪费方面 没有系统性差异。

家庭特征层面，家庭人口规模虽然对是否有食物浪费、食物浪费量和食物浪费率的 影响均为正, 但是没有通过显著性检验, 表明这一因素对大学生在高校食堂的食物浪费 行为没有关键影响, 这与针对居民家庭食物浪费行为的发现并不一致 ${ }^{[18]}$, 说明家庭消费 和外出公共就餐存在差异。与预期一致，家庭经济水平始终在 $1 \%$ 的显著性水平上正向促 进大学生的食物浪费，表现为家庭条件越优越，个体出现食物浪费的概率越高，食物浪 费量越大，食物浪费率也更高。这与关于居民家庭食物浪费的研究以及居民在餐馆就餐 时的食物浪费表现一致：经济条件越好食物浪费现象越严重 ${ }^{[2,252}$ 。

餐饮特征层面, 餐次对大学生是否有食物浪费并无显著影响, 表明个体吃午餐和吃 晚餐出现食物浪费的概率相差不大。然而, 是午餐还是晚餐会影响到食物浪费量和食物 浪费率。相对于晚餐，午餐时个体的食物浪费量更多，相应的食物浪费率也更高。就餐 持续时间没有通过显著性检验，表明这一因素对大学生食堂就餐的食物浪费行为没有关 键影响。一起就餐人数虽然对个体是否有食物浪费没有影响, 但是能够显著提升个体食 
物浪费量和食物浪费率, 表明集体行 动会加剧食物浪费。与预期一致, 餐 前饭菜重量显著正向促进大学生的食 物浪费行为, 表现为个体购置的饭菜 越多, 越可能出现食物浪费现象, 浪 费的食物越多，食物浪费率更高 ${ }^{[51]}$ 。 餐盘形式被一些研究证实会影响到高 校食堂的食物浪费 ${ }^{[24]}$, 本文发现, 这 一变量对个体是否有食物浪费没有影 响, 但是确实会显著影响个体的食物 浪费量和食物浪费率。相对于分装餐 盘, 合成餐盘会带来更多的食物浪费 量和更高的食物浪费率。既往研究表 明，个体对饮食的主观评价与食物浪 费行为密切相关 ${ }^{[23]}$, 本文再次印证了 这一判断。发现个体对饭菜满意度越 高, 出现食物浪费的概率更低, 相应 的食物浪费量和食物浪费率也更低。

区域特征层面，是否东部地区对 个体是否有食物浪费行为没有显著影 响, 说明相对于西部地区, 东部地区 大学生出现食物浪费的概率没有显著 差异。但相对于西部地区，东部地区 大学生表现出更高的食物浪费量和食 物浪费率，说明经济发达地区的个体 确实有着更为严重的食物浪费现象。 是否中部地区则对个体是否有食物浪 费、食物浪费量和食物浪费率均没有 显著性影响，说明相对于西部地区大 学生, 中部地区大学生的食物浪费行 为没有差异。

\section{3 作用机制检验与进一步分析}

\subsection{1 作用机制检验}

上述研究发现在高校食堂就餐 时, 南方籍贯大学生确实比北方籍贯 大学生更浪费食物。然而, 这只能说 明一个基本事实，却没有揭示南方人 为什么比北方人更浪费食物。一些跨 国研究表明，饮食文化差异是导致不 同国度居民食物浪费的根本原因 ${ }^{[38]}$ 。
表 4 南北方籍贯与食物浪费行为

Table 4 Correlation between North-South and food waste behavior

\begin{tabular}{|c|c|c|c|}
\hline 变量 & 是否有食物浪费 & 食物浪费量 & 食物浪费率 \\
\hline \multirow[t]{2}{*}{ 南北方籍贯 } & $0.065^{* *}$ & $17.102^{* * * *}$ & $3.450^{* * *}$ \\
\hline & $(0.031)$ & $(2.057)$ & $(0.407)$ \\
\hline \multirow[t]{2}{*}{ 性别 } & $-0.263^{* * *}$ & $-29.554^{* * *}$ & $-5.947^{* * *}$ \\
\hline & $(0.034)$ & $(2.187)$ & $(0.433)$ \\
\hline \multirow[t]{2}{*}{ 年龄 } & 0.005 & 0.733 & 0.186 \\
\hline & $(0.009)$ & $(0.631)$ & $(0.125)$ \\
\hline \multirow[t]{2}{*}{ 学历 } & $-0.147^{* * *}$ & $-7.643^{* *}$ & $-2.004^{* * *}$ \\
\hline & $(0.056)$ & $(3.730)$ & $(0.739)$ \\
\hline \multirow[t]{2}{*}{$B M I$ 指数 } & $-0.034^{* * *}$ & $-2.472^{* * *}$ & $-0.479^{* * *}$ \\
\hline & $(0.006)$ & $(0.387)$ & $(0.077)$ \\
\hline \multirow[t]{2}{*}{ 是否独生子女 } & 0.007 & 0.612 & 0.222 \\
\hline & $(0.043)$ & $(2.832)$ & $(0.561)$ \\
\hline \multirow[t]{2}{*}{ 民族 } & -0.081 & 2.833 & 0.449 \\
\hline & $(0.053)$ & $(3.366)$ & $(0.666)$ \\
\hline \multirow[t]{2}{*}{ 接触节粮宣传的频率 } & $-0.057^{*}$ & $-5.048^{* *}$ & $-0.843^{* *}$ \\
\hline & $(0.031)$ & $(2.058)$ & $(0.408)$ \\
\hline \multirow[t]{2}{*}{ 家庭人口规模 } & 0.028 & 2.883 & 0.457 \\
\hline & $(0.040)$ & $(2.615)$ & $(0.518)$ \\
\hline \multirow[t]{2}{*}{ 家庭经济水平 } & $0.114^{* * *}$ & $6.648^{* * *}$ & $1.377^{* * *}$ \\
\hline & $(0.021)$ & $(1.380)$ & $(0.273)$ \\
\hline \multirow[t]{2}{*}{ 餐次 } & 0.022 & $4.552^{* *}$ & $0.778^{*}$ \\
\hline & $(0.031)$ & $(2.043)$ & $(0.404)$ \\
\hline \multirow[t]{2}{*}{ 就餐持续时间 } & 0.131 & 1.760 & 0.402 \\
\hline & $(0.028)$ & $(1.796)$ & $(0.356)$ \\
\hline \multirow[t]{2}{*}{ 一起就餐人数 } & 0.029 & $2.871^{* *}$ & $0.512^{* *}$ \\
\hline & $(0.020)$ & $(1.300)$ & $(0.257)$ \\
\hline \multirow[t]{2}{*}{ 餐前饭菜重量 } & $0.001^{* * *}$ & $0.143^{* * *}$ & $0.006^{* * *}$ \\
\hline & $(0.000)$ & $(0.007)$ & $(0.001)$ \\
\hline \multirow[t]{2}{*}{ 餐盘形式 } & -0.017 & $3.642^{*}$ & $0.823^{*}$ \\
\hline & $(0.033)$ & $(2.146)$ & $(0.425)$ \\
\hline \multirow[t]{2}{*}{ 饭菜口味满意度 } & $-0.185^{* * *}$ & $-19.699^{* * *}$ & $-3.694^{* * *}$ \\
\hline & $(0.027)$ & $(1.713)$ & $(0.340)$ \\
\hline \multirow[t]{2}{*}{ 东部 } & 0.036 & $13.596^{* * *}$ & $2.596^{* * *}$ \\
\hline & $(0.037)$ & $(2.395)$ & $(0.474)$ \\
\hline \multirow[t]{2}{*}{ 中部 } & -0.002 & -2.812 & -0.616 \\
\hline & $(0.041)$ & $(2.713)$ & $(0.537)$ \\
\hline \multirow[t]{2}{*}{ 常数 } & $0.752^{* * *}$ & 28.246 & $16.001^{* * *}$ \\
\hline & $(0.264)$ & (17.664) & $(3.499)$ \\
\hline \multirow[t]{2}{*}{ sigma } & - & $87.294^{* * *}$ & $17.317^{* * *}$ \\
\hline & & $(0.829)$ & $(0.165)$ \\
\hline LR chi $^{2}$ & $416.03^{* * *}$ & $946.49^{* * * *}$ & $608.68^{* * * *}$ \\
\hline Pseudo $R^{2}$ & 0.045 & 0.012 & 0.011 \\
\hline 观测值 & 8157 & 8157 & 8166 \\
\hline
\end{tabular}

注: ${ }^{* * *}$ 、** “分别表示 $1 \% 、 5 \% 、 10 \%$ 的显著性水平，括号内 为标准误，下同。 
受此启发, 本文猜测地域饮食文化不同或许是造成中国南方人和北方人差异化食物浪费 表现的关键所在。由于地理和气候的差异，中国北方主要种植小麦，而中国南方主要种 植水稻。这也导致了南方占主导地位的是 “饭菜” 饮食模式，北方则形成了 “粉面” 的 主流饮食模式。因此，南北方的主流饮食模式差异能够很好地反映南北方的饮食文化差 异。那么, 体现南北方饮食文化差异的主流饮食模式是否是南北方人差异化食物浪费表 现的主要原因呢? 为验证这一可能的作用机制，本文引入高校所在的南北方区位来显示 南北方主流饮食模式。之所以选择高校的南北方区位来显示饮食模式差异，是考虑到高 校提供的公共餐饮有着很强的地域特色, 日常餐饮供给中很难摆脱本地主流饮食模式的 影响。进而使用中介效应模型对这一作用机制予以验证 ${ }^{[3]]}$, 检验结果如表 5 所示。

表 5 南北饮食模式的作用机制检验：中介效应模型

Table 5 Test of the mechanism of action of the North-South diet model: Mediating effect model

\begin{tabular}{|c|c|c|c|c|c|}
\hline 变量 & $\begin{array}{c}\text { (1) } \\
\text { 南北方区位 }\end{array}$ & $\begin{array}{c}\text { (2) } \\
\text { 是否有食物浪费 }\end{array}$ & $\begin{array}{c}\text { (3) } \\
\text { 南北方区位 }\end{array}$ & $\begin{array}{c}\text { (4) } \\
\text { 食物浪费量 }\end{array}$ & $\begin{array}{c}(5) \\
\text { 食物浪费率 }\end{array}$ \\
\hline \multirow[t]{2}{*}{ 南北方籍贯 } & $1.526^{* * * *}$ & -0.037 & $1.119^{* * *}$ & $5.870^{* *}$ & $1.186^{* *}$ \\
\hline & $(0.033)$ & $(0.037)$ & $(0.023)$ & $(2.434)$ & $(0.482)$ \\
\hline \multirow[t]{2}{*}{ 南北方区位 } & - & $0.200^{* * *}$ & - & $21.096^{* * *}$ & $4.257^{* * *}$ \\
\hline & & $(0.038)$ & & $(2.472)$ & $(0.489)$ \\
\hline \multirow[t]{2}{*}{ 常数 } & $0.545^{* *}$ & 0.635 & $0.377^{* *}$ & 15.545 & 13.411 \\
\hline & $(0.277)$ & $(0.265)$ & $(0.177)$ & $(17.646)$ & (3.494) \\
\hline 其他变量 & 已控制 & 已控制 & 已控制 & 已控制 & 已控制 \\
\hline \multirow[t]{2}{*}{ sigma } & - & - & 0.769 & 86.870 & 17.228 \\
\hline & & & $(0.010)$ & $(0.824)$ & $(0.164)$ \\
\hline LR $\mathrm{chi}^{2}$ & $2993.28^{* * *}$ & $444.56^{* * *}$ & $3261.42^{* * *}$ & $1019.15^{* * * *}$ & $684.24^{* * *}$ \\
\hline Pseudo $R^{2}$ & 0.266 & 0.048 & 0.196 & 0.013 & 0.012 \\
\hline 观测值 & 8180 & 8157 & 8180 & 8157 & 8166 \\
\hline
\end{tabular}

列（1）中，南北方籍贯通过了显著性水平检验，列（2）中南北方籍贯变的不再显 著，而指示南北方主流饮食模式差异的高校所在南北方区位通过了 $1 \%$ 显著性水平检验。 因此，可以判断南北方主流饮食模式是一个完全中介变量。即南方人比北方人更可能出 现食物浪费，确实是因为南方人和北方人的主流饮食模式有所不同。相对于北方的 “粉 面” 饮食模式，南方的 “饭菜” 饮食模式会增加个体食物浪费的概率。

同理，由于列（3）中南北方籍贯通过了 $1 \%$ 的显著性水平检验; 列（4）和列（5） 中的南北方籍贯通过了 $5 \%$ 的显著性水平检验，且高校所在南北方区位在 $1 \%$ 的显著性水 平正向促进食物浪费量和食物浪费率。因此可以判断出，南北方主流饮食模式是一个部 分中介变量。而且不难计算出, 在 “南北方籍贯一南北方主流饮食模式一食物浪费量” 和 “南北方籍贯一南北方主流饮食模式一食物浪费率” 的作用路径中，南北方饮食模式 分别能够解释个体 $80.09 \%$ 的食物浪费量差异和 $79.85 \%$ 的食物浪费率差异。即在高校食堂 就餐时, 南方籍贯大学生比北方籍贯大学生浪费更多食物, 食物浪费程度更高的一个关 键原因是：南北方的主流饮食模式有显著差异。

\subsection{2 进一步验证分析}

上述中介效应模型表明，南北方饮食模式是引致南方人比北方人更可能浪费食物的 
关键所在, 也是南方人比北方人浪费更多食物、浪费程度更重的主要原因。为进一步验 证上述结论的可靠性，基于另一种思路来予以检验。逻辑上，如果相对于北方的 “粉 面” 饮食模式，南方的 “饭菜” 饮食模式确实会引致更高的食物浪费概率，带来更多的 食物浪费量和更高的食物浪费率。那么, 相对于在南方高校就学的南方大学生, 在北方 高校就读的南方大学生的食物浪费情况会有所缓解。为此, 本文匹配了大学生的南北方 籍贯和所就读高校的南北方区位，形成了 “南方人在南方高校求学” “南方人在北方高校 求学” “北方人在南方高校求学” “北方人在北方高校求学” 四个类别。描述性分析发 现，“南方人在南方高校求学” 和 “北方人在南方高校求学” 的食物浪费现象更为普遍, 浪费量和浪费率也较高，而 “南方人在北方高校求学” 和 “北方人在北方高校求学” 的 食物浪费现象较少, 日常浪费量和浪费率也更低。进一步, 本文以“南方人在南方高校 求学” 为基准，在表 4 的基准方程之中引人了 “南方人在北方高校求学” “北方人在南方 高校求学” “北方人在北方高校求学” 三个虚拟变量, 拟合结果如表 6 所示。

结果显示，相对于 “南方人在南 方高校求学” 模式, “南方人在北方 高校求学” 通过了 $1 \%$ 显著性水平检 验，且影响方向为负。说明南方人在 北方求学确实会带来更低的食物浪费 概率，相应的食物浪费量会更少，食 物浪费率也更低。从而再次证实，南 北方的主流饮食模式是南方人和北方 人食物浪费表现分化的关键所在。与 此同时, “北方人在南方高校求学” 这一虚拟变量并没有通过显著性检 验, 说明相对于 “南方人在南方高校 求学”, 两种匹配模式下个体的食物 浪费表现没有显著差异。即北方人来 到南方后，没有比南方人表现出更低 的食物浪费概率、更少的食物浪费量 和更轻的食物浪费程度。这从另一层 面也证实，南方人和北方人的浪费行

表 6 进一步验证

Table 6 Further verification

\begin{tabular}{lccc}
\hline 变量 & 是否有食物浪费食物浪费量 & 食物浪费率 \\
\hline 南北方籍贯 & $0.179^{*}$ & $22.048^{* * *}$ & $3.557^{* * *}$ \\
& $(0.094)$ & $(6.319)$ & $(1.255)$ \\
南方人在北方求学 & $-0.176^{* * *}$ & $-18.168^{* * *}$ & $-3.762^{* * *}$ \\
& $(0.051)$ & $(3.348)$ & $(0.662)$ \\
北方人在南方求学 & 0.030 & 12.300 & 1.171 \\
& $(0.110)$ & $(7.372)$ & $(1.464)$ \\
北方人在北方求学 & 0.107 & $-0.290^{* *}$ & $-0.958^{* *}$ \\
& $(0.100)$ & $(6.737)$ & $(1.338)$ \\
常数 & $0.566^{* *}$ & 16.412 & $14.715^{* * *}$ \\
& $(0.285)$ & $(18.987)$ & $(3.765)$ \\
\hline 其他变量 & 已控制 & 已控制 & 已控制 \\
\hline sigma & - & $86.967^{* * * *}$ & $17.258^{* * *}$ \\
& & $(0.825)$ & $(0.164)$ \\
\hline LR chi ${ }^{2}$ & $438.88^{* * * *}$ & $997.89^{* * *}$ & $655.72^{* * *}$ \\
\hline Pseudo $R^{2}$ & 0.047 & 0.013 & 0.012 \\
\hline 观测值 & 8157 & 8157 & 8166 \\
\hline
\end{tabular}
为没有天然差异。之所以呈现南方人比北方人更浪费食物的事实状态，其症结是南北方 的主流饮食模式有显著差异，即南方的 “饭菜” 饮食模式确实会比北方的 “粉面” 饮食 模式带来更多的食物浪费。

\section{3 结论与讨论}

当前, 食物浪费现象已经成为一项世界性议题，引起了世界各国的广泛关注。中国 虽仍然是一个发展中国家，但是食物浪费现象非常突出。中国居民家庭和餐饮业的食物 浪费已经得到部分学者关注，但是还鲜有文献涉及公共食堂领域的食物浪费。中国是世 界上在校大学生数量最多的国家, 且大学生在食堂就餐时进行食物浪费的现象相当普 
遍，导致高校中的食物浪费情况十分严重。本文重点对中国高等院校公共食堂的食物浪 费进行了研究。研究视角方面, 不同于以往成果, 本文重点从南北地域差异视角解读了 个体的食物浪费行为, 重点考察了南方大学生和北方大学生的食物浪费表现及其背后的 作用机制。研究发现：在高校食堂就餐时，南北方籍贯显著影响个体食物浪费行为，相 对北方大学生, 南方大学生会更浪费食物。南方大学生不仅有着更高的食物浪费概率, 而且餐次食物浪费量更多, 餐次食物浪费率也更高。相对于北方人, 南方人出现食物浪 费的概率平均要高 2.05 个百分点, 平均每餐食物浪费量会多 $17.10 \mathrm{~g}$, 平均每餐的食物浪 费率要高 3.45 个百分点。为揭示南方大学生比北方大学生更浪费食物的原因所在, 本文 引人中介效应模型，探索了南北方主流饮食模式是否是南北方大学生差异化食物浪费表 现的症结所在。检验结果显示，主流饮食模式不同是南北方大学生食物浪费行为表现差 异的关键所在。即北方的 “粉面” 饮食模式导致了北方大学生有更低的食物浪费概率, 更少的食物浪费量，更低的食物浪费率；南方的 “饭菜” 饮食模式引致了南方大学生更 可能浪费食物，浪费了更多食物，食物浪费程度更重。

为进一步验证上述判断, 本文还匹配了大学生的南北方籍贯和求学高校所在的南北 方区位, 并以 “南方大学生在南方高校求学” 模式为基准, 发现 “南方大学生在北方高 校求学” 模式下，个体有着更低的食物浪费概率、食物浪费量和食物浪费率。但 “北方 学生在南方高校求学” 模式下，个体没有显示出较低的粮食浪费概率、食物浪费量和食 物浪费率。从而稳健的证实, 不是南方人有天然的浪费倾向, 而是南方和北方的主流饮 食模式不同, 导致了南方大学生比北方大学生更浪费食物。因此, 本文提供了一国之内 不同地域个体因为饮食文化不同导致食物浪费有较大差异的经验证据，从而丰富了本领 域的研究。

当然，本文的目标并不是为了证明南方人还是北方人更浪费食物，也不是为了说明 北方的 “粉面” 饮食模式比南方的 “饭菜” 饮食模式更优越, 而是试图呈现一个客观事 实：即相对而言，南方的饮食模式会导致更多的食物浪费。但南北方主流饮食模式是历 史、文化、气候、地理等多因素共同影响下的特定产物，有自身的合理性，很难进行系 统性变革, 也不可能为了更多节省食物就要求南方人更多地采纳粉面为主的饮食模式。 但令人鼓舞的是，当前南北方的饮食模式出现了日渐趋同的现象，南方人也越来越多的 吃面粉, 这可能有助于减少食物浪费。当然, 是否如此还有待后续研究予以追踪。

基于本文发现，现阶段需要从以下几个层面重点关注和管理高校食堂中的食物浪费 现象, 从而减少年轻人群体的浪费。第一, 要重点关注男性、本科生、 $B M I$ 指数较高个 体产生的食物浪费, 研究他们更浪费食物的深层次原因。考虑到接触节粮宣传的频率越 高, 越有助于个体节约粮食, 要进一步加大这一类宣传的力度。第二, 当个体家庭条件 越好时, 相应的食物浪费越突出。因此, 要重点关注这一类学生的食堂消费行为, 探寻 如何有效缓解和减少这一群体的食物浪费。第三, 餐饮消费属性特征对个体食物浪费有 十分关键的影响。鉴于餐次、一起就餐人数和餐盘形式能有效影响个体的浪费量和浪费 率, 要重点关注晚餐中的食物浪费现象, 探索如何减少多人就餐时产生的食物浪费, 并 更多地应用分装餐盘，提升高校食堂中这一类型餐盘的使用占比。更重要的，鉴于餐前 饭菜重量和饭菜口味满意度对个体食物浪费有十分显著的影响, 为了减少食物浪费, 应 推出小份饭菜供学生选择, 既满足消费多样性需求, 也有助于减少购买过量食物带来的 
浪费; 同时要加强高校食堂菜品口味的提升管理, 加强和学生的沟通, 推出受青年人欢 迎的美味菜品, 减少口味不满意带来的食物浪费。

本文还有一些不足之处，首先，本文只是证实了南方 “饭菜”饮食模式相对北方的 “面粉” 饮食模式，会引致更多和更严重的浪费食物，但为何前者比后者更浪费食物，则 有待进一步的调查与分析。其次，本文只是初步从南北差异视角来解读不同区域个体的 食物浪费行为分化，但是中国北方诸多省份的饮食也有差异，南方各省的饮食差异则更 大，比如八大菜系中有七大菜系是南方省份的，很多南方省份有自己的餐饮特色。因 此, 后续可以进一步细化, 研究不同省份的饮食差异如何影响个体的食物浪费表现。再 次, 本文涉及的南方大学生和北方大学生的食物浪费行为差异, 仅仅是高校食堂场所就 餐的青年大学生，其他场所如家庭、餐馆等是否呈现类似规律，或者更为一般的南北方 人而非大学生群体的食物浪费行为是否有类似差异，仍然有待后续研究子以跟进。

\section{参考文献(References):}

[1] 从农业文化遗产保护看零饥饿目标实现. http://f.china.com.cn/2018-10/16-241:evelopment Research,2012/conten241:evelopment Research,2012t_66458997.htm, 2018-10-16. [To achieve the goal of zero hunger from the perspective of agricultural cultural heritage protection. http://f.china.com.cn/2018-10/16- 241:evelopment Research,2012/conten241:evelopment Research,2012t_66458997.htm, 2018-10-16.]

[2] 全球挨饿人口持续增加, 2030 年消除饥饿愈发艰难. http://www.cutv.com/guoji/2018-9-12/1536716409689.shtml, 2018-09-12. [The number of hungry people in the world continues to increase, and it will be more and more difficult to eradicate hunger in 2030. http://www.cutv.com/guoji/2018-9-12/1536716409689.shtml, 2018-09-12.]

[3] LUNDQVIST J, DE FRAITURE C, MOLDEN D. Saving water: From field to fork-curbing losses and wastage in the food chain. Stockholm: Stockholm International Water Institute, 2008: 1-36.

[4] LIPINSKI B, HHANSON C, LOMAX J, et al. Reducing Food Loss and Waste. Washington D C: World Resources Institute Working Paper, 2013: 1-40.

[5] 高利伟, 成升鬼, 曹晓昌, 等. 食物损失和浪费研究综述及展望. 自然资源学报, 2015, 30(3): 523-536. [GAO L W, CHENG S K, CAO X C, et al. Review and prospect of food loss and waste research. Journal of Natural Resources, 2015, 30(3): 523-536.]

[6] 曹芳芳, 黄东, 俊峰武, 等. 小麦收获损失及其主要影响因素: 基于 1135 户小麦种植户的实证分析. 中国农村观察, 2018, (2): 75-87. [CAO F F, HUANG D, JUN F W, et al. Wheat harvest loss and its main influencing factors: An empirical analysis based on 1135 wheat growers. China Rural Survey, 2018, (2): 75-87.]

[7] 王灵恩, 成升鬼, 刘刚, 等. 中国食物浪费研究的理论与方法探析. 自然资源学报, 2015, 30(5): 715-724. [WANG L E, CHENG S K, LIU G, et al. Analysis of theories and methods of food waste research in China. Journal of Natural Resources, 2015, 30(5): 715-724.]

[8] 成升鬼, 高利伟, 徐增让, 等. 对中国餐饮食物浪费及其资源环境效应的思考. 中国软科学, 2012, (7): 106-114. [CHENG S K, GAO L W, XU Z R, et al. Food waste in catering industry and its impacts on resources and environment in China. China Soft Science, 2012, (7): 106-114.]

[9] BAI J, ZHANG C, WAHL T. Dining out, the missing food consumption in China. Applied Economics Letters, 2016, 23 (15): 1084-1087.

[10] 江金启, 黄琬真, 王振华. 中国家庭食物浪费的规模估算及决定因素分析. 农业技术经济, 2018, (9): 88-99. [JIANG J Q, HUANG W Z, WANG Z H. Analysis of the scale and determinants of household food waste in China. Agricultural Technology Economy, 2018, (9): 89-99.]

[11] GJERRIS M, GAIANI S. Household food waste in Nordic countries: Estimations and ethical implications. Etikki praksis-Nordic Journal of Applied Ethics, 2013, 1: 6-23.

[12] PORPINO G. Household food waste behavior: Avenues for future research. Journal of the Association for Consumer Research, 2016, 1(1): 41-51. 
[13] 张盼盼, 白军飞, 成升鬼, 等. 信息干预是否影响食物浪费? 基于餐饮业随机干预试验. 自然资源学报, 2018, 33(8): 1439-1450. [ZHANG P P, BAI J F, CHENG S K, et al. Does information intervention affect food waste? Based on random intervention trials in the catering industry. Journal of Natural Resources, 2018, 33(8): 1439-1450.]

[14] 廖芬, 青平, 孙山, 等. “殊途同归”: 沟通方式对食物浪费行为的影响机制分析. 中国农村经济, 2018, (5): 1-17. [LIAO F, QING P, SUN S, et al. "Achieve the same path": Analysis of the influence mechanism of communication methods on food waste behavior. China Rural Economy, 2018, (5): 1-17.]

[15] WANG P, LIU Q, QI Y. Factors Influencing sustainable consumption behaviors: A survey of the rural residents in China. Journal of Cleaner Production, 2014, 63: 152-165.

[16] VISSCHERS V H M, WICKLI N, SIEGRIST M. Sorting out food waste behavior: A survey on the motivators and barriers of self-reported amounts of food waste in households. Journal of Environmental Psychology, 2016, 45: 66-78.

[17] KOIVUPURO H K, HARTIKAINEN H, SILVENNOINEN K, et al. Influence of socio-demographical, behavioral and attitudinal factors on the amount of avoidable food waste generated in Finnish households. International Journal of Consumer Studies, 2012, 36(2): 183-191.

[18] SONG G, LI M, SEMAKULA H M, ZHANG S. Food consumption and waste and the embedded carbon, water and ecological footprints of households in China. Science of the Total Environment, 2015, 529: 191-197.

[19] HAMILTON C, DENNISS R, BAKER D G. Wasteful Consumption in Australia. Canberra: Australia Institute, 2005: 146.

[20] SEGRE A, FALASCONI L, POLITANO A, et al. Background paper on the economics of food loss and waste. Rome: Food and Agriculture Organization of the United Nations, 2014: 1-83.

[21] KAUKONEN K M, BAILEY M, SUZUKI S, et al. Mortality related to severe sepsis and septic shock among critically Ill patients in Australia and New Zealand, 2000-2012. The Journal of the American Medical Association, 2014, 311(13): 1308-1316.

[22] 王禹, 许世卫, 李哲敏, 等. 城市居民在外就餐食物浪费影响因素实证研究. 中国农业资源与区划, 2018, 39(9): 199204. [WANG Y, XU S W, LI Z M, et al. Empirical study on the factors affecting food waste in urban residents. Chinese Journal of Agricultural Resources and Regional Planning, 2018, 39(9): 199-204.]

[23] 孙中叶. 解读粮食安全问题的新视角: 开源节流并举: 兼论河南家庭粮食消费损失现状及对策. 河南工业大学学 报: 社会科学版, 2009, 5(3): 1-4. [SUN Z Y. A new perspective on interpreting food security issues: Opening up and savings together: On the status quo and countermeasures of household food consumption loss in Henan province. Journal of Henan University of Technology: Social Science Edition, 2009, 5(3): 1-4.]

[24] MARLETTE M A, TEMPLETON S B, PANEMANGALORE M. Food type, food preparation, and competitive food purchases impact school lunch plate waste by sixth-grade students. Journal of the American Dietetic Association, 2005, 105(11): 1779-1782.

[25] ADAMS M A, PELLETIER R L, ZIVE M M, et al. Salad bars and fruit and vegetable consumption in elementary schools: A plate waste study. Journal of the American Dietetic Association, 2005, 105(11): 1789-1792.

[26] BUZBY J C, HYMAN J. Total and per capital value of food loss in the United States. Food Policy, 2012, 37(5): 561570.

[27] MOULT J A, ALLAN S R, HEWITT C N, et al. Greenhouse gas emissions of food waste disposal options for UK retailers. Food Policy, 2018, 77: 50-58.

[28] BARTON A D, BEIGG C L, MACDONALD I A, et al. High food wastage and low nutritional intakes in hospital patients. Clinical Nutrition, 2000, 19(6): 445-449.

[29] ZAKIAH L, SAIMY I, MAIMUNCH A. Plate waste among hospital inpatients. Malaysian Journal of Public Health Medicine, 2005, 5(2): 19-24.

[30] WHITEHAIR K J, SHANKLIN C W, BRANNON L A. Written messages improve edible food waste behaviors in a University dining facility. Journal of the Academy of Nutrition and Dietetics, 2013, 113(1): 63-69.

[31] SCHAUBROECK T, CEUPPENS S, LUONG A D, et al. A pragmatic framework to score and inform about the environmental sustainability and nutritional profile of canteen meals: A case study on a university canteen. Journal of Cleaner Production, 2018, 187: 672-686. 
[32] 胡越, 周应恒, 韩一军, 等. 减少食物浪费的资源及经济效应分析. 中国人口·资源与环境, 2013, 23(12): 150-155. [HU Y, ZHOU Y H, HAN Y J, et al. Analysis of resources and economic effects of reducing food waste. China Population, Resources and Environment, 2013, 23(12): 150-155.]

[33] WANG L, LIU G, LIU X, et al. The weight of unfinished plate: A survey based characterization of restaurant food waste in Chinese cities. Waste Management, 2017, 66: 3-12.

[34] 张丹, 成升魁, 高利伟, 等. 城市餐饮业食物浪费的生态足迹: 以北京市为例. 资源科学, 2016, 38(1): 10-18. [ZHANG D, CHENG S K, GAO L W, et al. Ecological footprint of food waste in urban catering industry: Taking Beijing as an example. Resources Science, 2016, 38(1): 10-18.]

[35] 张丹, 成升魁, 高利伟, 等. 城市餐饮业食物浪费碳足迹: 以北京市为例. 生态学报, 2016, 36(18): 5937-5948. [ZHANG D, CHENG S K, GAO L W, et al. Food waste carbon footprint in urban catering industry: Taking Beijing as an example. Acta Ecologica Sinica, 2016, 36(18): 5937-5948.]

[36] 张丹, 伦飞, 成升鬼, 等. 城市餐饮食物浪费的磷足迹及其环境排放: 以北京市为例. 自然资源学报, 2016, 31(5): 812-821. [ZHANG D, LUN F, CHENG S K, et al. Phosphorus footprint of urban food waste and its environmental emissions: Taking Beijing as an example. Journal of Natural Resources, 2016, 31(5): 812-821.]

[37] PAINTER K, THONDHLANNA G, KUA H W. Food waste generation and potential interventions at Rhodes University, South Africa. Waste Management, 2016, 56: 491-497.

[38] PINTO R S, DOS SANTOS PINTO R M, MELO F F S, et al. A simple wariness campaign to promote food waste reduction in a University Canteen. Waste Management, 2018, 76: 28-38.

[39] LORENZ B A S, HARTMANN M, LANGEN N. What makes people leave their food? The interaction of personal and situational factors leading to plate leftovers in canteens. Appetite, 2017, 116: 45-56.

[40] SECONDI L, PRINCIPATO L, LAURETI T. Household food waste behavior in EU-27 countries: A multilevel analysis. Food Policy, 2015, 56: 25-40.

[41] PARFITT J, BARTHEL M, MACNAUGHTON S. Food waste within food supply chains: Quantification and potential for change to 2050. Philosophical Transactions of the Royal Society of London B: Biological Sciences, 2010, 365 (1554): 3065-3081.

[42] TALHELM T, ZHANG X, OISHI S, et al. Large-scale psychological differences within China explained by rice versus wheat agriculture. Science, 2014, 344(6184): 603-608.

[43] MARTINS M L, CUNHA L M, RODRIGUES S S P, et al, Determination of plate waste in primary school lunches by weighing and visual estimation methods: A validation study. Waste Management, 2014, 34(8): 1362-1368.

[44] DERQUI B, FERNANDEZ V. The opportunity of tracking food waste in school canteens: Guidelines for self-assessment. Waste Management, 2017, 69: 431-444.

[45] BOSCHINI M, FALASCONI L, GIORDANO C, et al. Food waste in school canteens: A reference methodology for large-scale studies. Journal of Cleaner Production, 2018, 182: 1024-1032.

[46] LORENZ B A S, HARTMANN M, LANGEN N. What makes people leave their food: The interaction of personal and situational factors leading to plate leftovers in canteens. Appetite, 2017, 116: 45-56.

[47] BUZBY J C, GUTHRIE J F. Plate Waste in school nutrition programs. The Journal of Consumer Affairs, 2002, 36(2): 220-238.

[48] THYBERG K L, TONJES D J. Drivers of food waste and their implications for sustainable policy development. Resources, Conservation and Recycling, 2016, 106: 110-123.

[49] YOUNG W, RUSSELL S V, ROBINSON C A, et al. Can social media be a tool for reducing consumers' food waste: A behavior change experiment by a UK retailer. Resources, Conservation and Recycling, 2017, 117: 195-203.

[50] PARIZEAU K, VON MASSOW M, MARTIN R. Household-level dynamics of food waste production and related beliefs, attitudes, and behaviors in Guelph, Ontario. Waste Management, 2015, 35: 207-217.

[51] 王灵恩, 成升鬼, 穆松林, 等. 拉萨市餐饮食物消费实证研究. 开发研究, 2012, (4): 147-152. [WANG L E, CHENG S K, MU S L, et al. Empirical research on catering food consumption in Lhasa. Development Research, 2012, (4): 147152.]

[52] GUSTAVSON J, CEDERBERG C, SONESSON U, et al. Global food losses and food waste. Rome: FAO, 2011: 1-24. 


\title{
Research on individual food waste behavior from the perspective of North-South differences: \\ Take the dining situation of students in university canteens as an example
}

\author{
QIAN Long ${ }^{1}$, LI Feng ${ }^{1}$, QIAN Zhuang ${ }^{1}$, WANG Ling-en² \\ (1. Center for Food Security and Strategic Research, Nanjing University of Finance and Economics, Nanjing \\ 210003, China; 2. Institute of Geographic Sciences and Natural Resources Research, CAS, Beijing 100101,
} China)

\begin{abstract}
Eating habits has always been considered as an important factor in food waste. This paper takes 29 universities, all in different provincial-level regions, as study cases to explore the factors that affect students' wasting behavior. Based on a questionnaire survey of 9192 college students, this paper focuses on the impact of North-South differences on individual food waste behavior, and attempts to answer: Southerners and Northerners, who are more likely to waste food, who waste more food? The results show that compared with the northern native college students, the probability of food waste in the students of the southern region is higher, and the food waste and food waste rate of the students of the southern origins in the college cafeteria are higher than those of the northern students. In order to verify whether the difference in diet patterns between the "rice" model in the south and the "wheat" model in the north is the main reason for the above phenomenon, this paper confirms that the difference between the north and the south diet patterns is the key reason for the fact that the southerners waste more food than the northerners through the mediation effect model. This paper further matches the individual's North-South origin and the North-South position of the colleges and universities. Based on the mode of "Southern students studying in the South", we found that the "Southern tudents going to school in the North" mode has a lower probability of food waste, waste amount and food waste rate. However, under the "Northern students going to school in the South" mode, individuals did not have a low probability of food waste, the weight of waste and food waste rate. Therefore, this paper confirms that the southerners are more wasteful of food than the northerners. And it is proved that the mainstream diet pattern in the north and the south is the leading driving force to the difference in waste.
\end{abstract}

Keywords: food waste; college canteen; North-South difference; mainstream diet 